\title{
LES FORMES DU SAVOIR ET L'ÉDUCATION AUX IMAGES
}

\author{
Bernard Darras ${ }^{1}$
}

Après avoir tenté de découpler les antonymes "formel" et "informel" au profit d'une reconnaissance de leur spécificité, et d'une ouverture sur les savoirs "complexes", cet article abordera l'influence probable sur les performances aux tests d'intelligence des savoirs complexes dérivés de la fréquentation des images. Puis, en exploitant les théories issues de la psychologie, de la sémiotique cognitive et de la systémique, seront abordées les différentes formes de construction des savoirs nécessaires à la fabrication des images par les sujets novices.

\section{Savoirs formels et informels, propositions de découplage}

Dans les langues romanes et probablement dans bien d'autres, les constructions de l'absence, de l'opposé ou du contraire d'un terme se font couramment en utilisant des préfixes négatifs ou privatifs, voire plus simplement une négation. Si la syntaxe est économique,

1 Professeur à l'Université Paris I Panthéon-Sorbonne. Chercheur au Centre de Recherche sur l'Image. 
l'opération a un coût sémantique qui n'est pas négligeable. En effet, les mots ainsi formés conservent une dépendance sémantique forte avec le radical qu'ils nient ou contrarient, ce qui ne manque pas d'influencer leur signification. D'une certaine manière, la dérivée négative ou contraire renvoie toujours aux propriétés sémantiques du radical. Quand, de l'affirmation à la négation, la catégorie conceptuelle est vraiment continue, l'opération est sans dommage, en revanche s'il y a discontinuité, et plus encore s'il y a écart, l'utilisation du préfixe peut masquer une différence radicale et rendre opaque la relation entre les entités. En bonne économie terminologique, il importe donc ici d'examiner la paire formée par les antonymes "formel" et "informel" (ou non formel).

Étymologiquement le premier terme a servi à qualifier ce qui est déterminé par une forme ou son principe. Ses propriétés de base en sont la limite, l'organisation, le principe d'unité interne, la stabilité, la norme, la règle, et la reproductibilité. À l'inverse, est déclaré "informel" tout ce qui ne possède pas ces propriétés soit, l'illimité, l'inorganisé, l'instable, l'irrégulier et le non reproductible. Ensemble de propriétés dont les préfixes en $i r, i l$, ou in, ne fonctionnent pas seulement comme des indicateurs privatifs, mais aussi comme des marqueurs péjoratifs dans le contexte culturel dominant de la maîtrise, de la règle, du simple et de l'ordre.

Il nous semble donc qu'appliqué au domaine des savoirs, le couple formel et informel ne manque pas de qualifier, mais aussi de déqualifier et de disqualifier. Nous faisons ici l'hypothèse que la partition du domaine des savoirs par le couple "formel - informel" privilégie les propriétés formelles au détriment d'autres propriétés ; qu'elle réduit la question des savoirs à une de ses facettes et qu'elle néglige les autres ; enfin, qu'elle méconnaît ou sous estime les organisations complexes des savoirs. Ne serait-ce que sur ce point, on sait aujourd'hui reconnaître de l'organisation dans des systèmes dont la complexité est si grande qu'ils ont été longtemps considérés comme n'en ayant pas. Ce qui a permis d'enrichir considérablement la palette des modes d'organisation au-delà des formes simples, linéaires ou hiérarchiques.

En conséquence, on dira que les savoirs formels sont essentiellement concentrés autour des systèmes sémiotiques conventionnels et formalisés qui ont tendance à la fermeture. Le plus souvent, ils sont sensibles (ou résultent) des approches réductionnistes et analytiques et 
sont en conséquence particulièrement compatibles avec les médias linéaires, verbaux et textuels. D'où la préférence que leur accorde l'école qui est restée le domaine du logos.

Autre conséquence, on dira que les autres savoirs relèvent de domaines encore peu ou mal explorés, compris, ou valorisés.

Parmi eux figurent les domaines complexes, possédant un très grand nombre de variables, peu déterminés, dynamiques, peu prévisibles, soumis à de grandes variations et très sensibles à leurs environnements. On peut y placer quelques-uns de nos plus grands savoirs tels que : savoir vivre, savoir vivre ensemble, savoir communiquer, savoir parler, savoir voir, savoir apprendre, savoir être parent, savoir enseigner, etc. Ces savoirs construits dans des situations d'immersion sont particulièrement difficiles à réduire et à décomposer. Ils résistent à la description, et précisément à la formalisation. Ce faisant, ils échappent particulièrement aux modes de transmission les plus scolaires.

Par ailleurs, nous ne nous priverons pas du terme "informel", mais en accord avec nos conclusions sémantiques et pragmatiques, nous en réserverons l'emploi à tous les savoirs fragmentaires, inachevés, inaboutis, confus ou mal maîtrisés.

En conclusion, nous suggérons que l'étude soit déplacée vers les différentes formes du savoir, tout en évitant le piège de la simplification dualiste fondé sur les a priori et les habitudes scolaires. À cette occasion, il est probable que même les savoirs formels y gagneront en précision, diversité et complexité.

Après cette entrée en matière très générale, nous voudrions étudier un des domaines où la question de la forme des savoirs et celle de leurs apprentissages est particulièrement complexe, voire informelle.

On utilise généralement le terme d'image pour qualifier ce domaine, mais ici encore l'acception ordinaire est un peu étriquée et ne permet pas d'embrasser l'ensemble de l'univers des artefacts concernés. En qualité de signes possédant une dimension iconique forte, les images sont très souvent associées à d'autres systèmes de signes et constituent des ensembles complexes aux fonctions, finalités et utilisations multiples. On les rencontre particulièrement sous cette forme dans les schémas, les illustrations, l'audiovisuel et le multimédia. 
À la recherche d'études manifestant l'impact et les effets de ces univers d'images sur les fonctions supérieures de la pensée, je m'intéresserai ci-après aux récentes hypothèses tentant d'expliquer l'effet Flynn.

\section{Les savoirs construits à partir des univers d'images et leur impact sur les réponses aux tests d'intelligence}

Les études de l'évolution des performances aux tests standardisés convergent et reconnaissent une courbe ascendante des scores. Le découvreur de ce phénomène n'est pas un psychologue, mais un professeur de Sciences politiques inquiété par les interprétations abusives de ces tests. Professeur à l'Université d'Otago en NouvelleZélande, James R. Flynn a vu son nom associé à cet effet de croissance constante ${ }^{1}$. Cette croissance de plus de trois points de quotient intellectuel par décade s'observe avec tous les tests importants, pour toutes les classes d'âge, pour tous les groupes et dans tous les pays industrialisés. Plus encore, il apparaît que globalement les gains ne cessent de croître et que la tendance semble elle-même en voie d'accélération.

Aujourd'hui, les explications des causes de ce phénomène demeurent partiellement hypothétiques.

Bien évidemment, les pistes les plus évidentes ont été testées, mais on ne peut leur attribuer qu'une partie de la croissance. La meilleure nutrition, la croissance de la taille du corps et celle du cerveau, la plus longue scolarisation des populations, l'habituation aux tests, etc. ne sont que partiellement responsables de la croissance.

Dès ses premières études comparatives, Flynn a noté que les performances d'un groupe d'individus sont bien meilleures si on utilise des versions anciennes et non re-standardisées des tests ${ }^{2}$. La réciproque est aussi vraie, ainsi en est-il de l'abaissement du quotient intellectuel (Q.I.) des personnes âgées. Il a longtemps été attribué au

1 J.R. FLYNN, "The mean IQ of Americans : Massive gains 1932 to 1978", Psychological Bulletin, $\mathrm{n}^{\circ}$ 95, 1984, pp. 29-51.

2 J.R. FLYNN, "Massive IQ gains in 14 nations: What IQ tests really measure", Psychological Bulletin, $\mathrm{n}^{\circ}$ 101, 1987, pp. 171-191. 
vieillissement, alors qu'il n'est qu'un artefact. En effet, les études de Flynn montrent que les performances d'un sujet restent relativement stables dans le temps, ce qui se vérifie si le sujet est toujours testé avec la même version du test.

Le soupçon a donc porté sur les opérations de re-standardisation des tests et sur la sensibilité des tests aux changements d'environnement cognitif et de mode de vie.

Une plus grande attention portee aux constituants des tests a montré que les gains de Q.I. aux modules exploitant les connaissances liées au vocabulaire, à l'arithmétique et à la culture générale était en faible croissance, voire en stagnation. Dans l'ensemble, ce sont d'ailleurs les tests qui sont les plus dépendants des matières scolaires qui connaissent les plus faibles progressions.

En revanche, les tests les moins "verbaux" mesurent ou provoquent les plus grands gains de quotient intellectuel. C'est notamment le cas du test des Matrices Progressives de Raven ${ }^{1}$, qui est essentiellement fondé sur des résolutions de problèmes à partir de figures spatiales. Ce test de raisonnement, destiné à mesurer l'intelligence "fluide", a été longtemps considéré comme l'un des plus "indépendants" de la culture et de l'éducation. Or, il apparaît qu'à ce test les gains sont de près de six points de Q.I. par décade ${ }^{2}$, et de 15 points de déviation par génération ${ }^{3}$.

Dans la mesure où il est peu probable que l'intelligence des populations testées croisse aussi rapidement, l'indépendance culturelle de ce test est elle aussi contestée. Ainsi, pour Patricia Greenfield,

Les tests de Q.I. non verbaux sont, en fait, plus et non pas moins culturellement sensibles que les tests verbaux. (...) De fait, ces tests non verbaux ou de performance dépendent de leur propre langage de conventions visuelles. (...), même la terminologie "d'impact culturel réduit" est une appellation

1 J.C. RAVEN, J.H. CourT, \& J. RAVEN, Manual for Raven's progressive Matrices and Vocabulary Scales (section 3), Oxford, Oxford Psychologist Press, 1992.

2 J.R. FLYNN, "IQ Gains Over Time : Toward Finding the Cause", in U. NEISSER (ed.), The Rising Curve,Long-Term Gains in IQ and Related Measures, Washington (DC), Amrican Psychological Association, 1998.

3 J.R. FLYNN, (1987), op. cit, et J.R. FLYNN, "IQ gains over time", in R.J. STERNBERG (ed.), Encyclopedia of Human Intelligence, New York, Macmillan, 1994, pp. 617623. 
erronée pour des tests visuels comme les Matrices Progressives de Raven ${ }^{1}$.

Ce sont donc les changements dans l'univers visuel et spatial et leurs impacts sur la connaissance et les connaissances qui sont considérés par de nombreux auteurs comme les causes probables des gains de Q.I. aux tests. Ces gains résulteraient, en quelque sorte, d'une adaptation cognitive à la culture des artefacts visuels dont le développement a été considérable tout au long du vingtième siècle. C'est aussi ce que soupçonne Ulric Neisser ${ }^{2}$, qui écrit :

Il est donc possible qu'une forme particulière d'intelligence se soit effectivement développée au contact des médias visuels complexes. On pourrait la définir comme une aptitude à l'analyse visuelle. (...) Du coup, les paradoxes suscités par l'effet Flynn se dissipent. S'il s'agit de l'aptitude à l'analyse visuelle, nous sommes effectivement plus malins que nos grandsparents. Ce n'est pas vrai pour les autres aspects de l'intelligence ${ }^{3}$.

Si l'hypothèse se confirme, nous disposons d'une sorte d'instrument de mesure diachronique de l'impact du monde des artefacts visuels sur une partie de l'intelligence, ou -dans une perspective d'intelligences modulaires- sur les intelligences visuelles et spatiales.

Dans la mesure où ces gains ont été enregistrés avant la généralisation de la télévision, nous pouvons même avancer l'hypothèse qu'elle n'est pas la seule source de cette adaptation des compétences spécialisées.

En tout cas, il est probable que la prolifération des images fixes et animées, des graphes, organigrammes, idéogrammes, diagrammes et pictogrammes et autres schémas, ait laissé une trace sur les instruments de la psychométrie. On peut même conjecturer que l'impact des images interagies et des jeux vidéos influencera les gains à venir ${ }^{4}$.

En ce qui concerne notre réflexion, ces études montrent que tout un univers de compétences sémiotiques, d'habileté cognitive, et de

I P.M. GREENFIELD, "The Cultural Evolution of IQ", in U. NEISSER (ed.), The Rising Curve..., op. cit.

2 U. NEISSER, "Sommes-nous plus intelligents que nos grands-parents", La Recherche, $\mathrm{n}^{\circ}$ 309, 1998, pp. 46-52.

3 Voir aussi U. NEISSER (ed.), The Rising Curve..., op. cit.

4 D'après une enquête publiée sur le site <www.educnet.education.fr/langues/ sitelang/intro.htm>, en France, les jeunes de 8 ans à plus de 15 ans jouent en moyenne quatre heures par semaine sur leurs divers jeux vidéo électroniques. 
savoirs diffus, est acquis par l'immersion dans la culture visuelle et qu'il marque profondément, radicalement et durablement la connaissance et les compétences cognitives. Par rapport à notre problématique, il est intéressant de noter que ces savoirs n'appartiennent pas vraiment à ce qu'il est convenu d'appeler les savoirs formels. Bien au contraire, ils relèvent plutôt de la zone que -faute de mieux et en attendant un travail de discrimination et de catégorisation- nous appelons les savoirs complexes. Ces savoirs ont la particularité de ne pouvoir se décomposer, aussi s'acquièrent-ils généralement par l'immersion, l'imitation, les interactions multiples et la pratique.

L'accélération de la croissance des gains de Q.I. témoigne à la fois de l'importance qu'on accorde à ces dimensions lors de la restandardisation des tests, et de l'habileté croissante des sujets jeunes dans le traitement des informations visuo-spatiales, ainsi que dans la résolution des problèmes de ce type.

Les changements de l'environnement visuel sont même fortement soupçonnés de stimulation cognitive précoce puisque les gains de Q.I. sont notés chez les jeunes enfants.

Toutefois, même si la valeur moyenne des performances aux Matrices Progressives de Raven et aux modules similaires des tests mixtes se déplace de six points par décade, et même si toute la population testée semble emportée dans ce mouvement, la distribution en courbe de Gauss des performances manifeste la même disparité entre les sujets.

Ces savoirs et compétences ne sont donc pas également répartis dans la population. Il est donc possible de considérer que les tests évaluent simultanément les compétences à l'analyse visuelles, mais aussi la richesse ou les lacunes de la culture visuelle du sujet.

À l'ère de la vidéo et de la photo numériques, du scanneur, du traitement vectoriel ou bitmap de l'image, des images de synthèse, des images interagies, etc., ne faut-il pas considérer que les technologies récentes bouleversent radicalement la culture visuelle. En ce domaine, une des grandes nouveautés provient du fait qu'il est aujourd'hui aussi aisé de produire et de manipuler des images que d'en utiliser ${ }^{1}$.

1 B. Darras, Au commencement était l'image. Du dessin de l'enfant à la communication de l'adulte, Paris, ESF, 1996. B. Darras, "Multimédia et éducation à l'image", in B. DARras (ed.), Multimédia et Savoirs, Paris, L'Harmattan, 2000, pp. 143-156. B. DARRAS, "L'école face aux cultures des 
Nous aborderons précisément ce point dans la partie suivante.

\section{Fabrication des images et formes de l'apprentissage}

Engagés dans des recherches sur l'image depuis un peu plus de vingt ans, mes travaux ont été particulièrement intéressés par la question de leur fabrication et par celle qui concerne l'importance des images dans la pensée et la communication.

En ce domaine, les premières recherches se sont développées pendant la première moitié $\mathrm{du} \mathrm{XX}^{\mathrm{e}}$ siècle avant d'être synthétisées par Jean Piaget. Elles ont presque toutes abordé les images sous l'angle de la représentation, et ont privilégié les dimensions optiques et spatiales. En ce sens, la théorie de Piaget est dominée par une conception développementale, linéaire et unidirectionnelle. Bien qu'elle soit constructiviste, elle a privilégié le développement interne (dans le sujet) des compétences, en minorant l'impact de l'environnement et des autres sources d'apprentissage.

L'érosion progressive de cet ensemble théorique a favorisé l'éclosion de deux nouvelles orientations.

La première est très marquée par les approches sémiotiques et cognitives et s'intéresse particulièrement à la construction des figures. Elle privilégie la vocation communicationnelle des "écritures" en images, et décrit la formation des figures dans le contexte d'émergence et de régulation cognitive et sociale des processus de conceptualisation des savoirs.

La seconde est issue de différentes influences théoriques (sémiotique, systémique, théorie des genres, etc.) qui toutes portent leur attention sur l'organisation des différents systèmes de représentation. Elle débouche sur une approche des relations que ces systèmes entretiennent dans l'écosystème des images.

Ces approches théoriques plus récentes souscrivent à l'idée que les premiers apprentissages se réalisent par immersion dans le creuset des multiples occasions de construction des savoirs, et qu'il en résulte

médias", Résonances, "La lecture d'images", $\mathrm{n}^{\circ} 8,2001$, pp. 14-15. R. LA BORDERIE, "Éduquer par l'image/à l'image", Résonances, "La lecture d'images", $n^{\circ} 8,2001$, pp. 8-9. R. LA BORDERIE, Éducation à l'image et aux médias, Paris, Nathan, 1997. B. HÉBUTERNE-PoINSAC, L'image éducatrice, Paris, PUF, 2000. 
un ensemble de compétences minimales. Elles constatent que ces compétences sont globalement informelles et sous développées par rapport aux potentialités communicationnelles et expressives des systèmes d'image. Elles constatent aussi qu'elles sont sous-développées par rapport aux attentes, à la demande et aux besoins de communication, de création et d'expression des individus. Elles montrent enfin que le sous-développement et l'apprentissage inaccompli peuvent être compensés par des apprentissages spécifiques éventuellement plus formels.

Il n'est pas possible de résumer en quelques lignes l'ensemble de ces cadres théoriques, ce qui d'ailleurs nous éloignerait des objectifs de cet article. Aussi, cette courte présentation se limitera-t-elle à traiter de la problématique des savoirs formels, informels, et complexes dans la fabrication des images.

\subsection{Théorie de la représentation et psychologie du développement}

Dès la fin du XIXe siècle et au début du $\mathrm{XX}^{\mathrm{e}}$ siècle, les premiers théoriciens qui ont considéré la production graphique des enfants -mais aussi celles des hommes "préhistoriques" et des "primitifs"ont résolument opté pour une approche évolutionniste profondément marquée par les enjeux visuels et spatiaux.

Reprenant les phases du développement proposées par Luquet, Jean Piaget a confirmé ses conceptions. Il les a intégrées dans son étude du développement de l'intelligence en insistant tout particulièrement sur la construction des savoirs spatiaux ${ }^{1}$.

La représentation figurative était évaluée en fonction de "la réalité visible" considérée à la fois comme référence et mesure. En conséquence, les transformations observées dans le processus représentatif manifestaient la tendance "naturelle", de l'humanité et de chaque humain à tendre vers plus de réalisme.

Si le savoir pouvait être considéré comme formellement délimité par l'expérience de la réalité, d'une part, et l'expérience des performances de l'illusion de réalité d'autre part, les modalités d'accès à ce

1 J. PIAGET, B. INHELder, La représentation de l'espace, chez l'enfant, Paris, PUF, 1948. 
savoir(-faire illusion) étaient considérées selon deux approches complètement étrangères.

La première était presque exclusive et s'intéressait au processus de développement des compétences dans le cadre du développement de l'intelligence. À ce titre la production d'image était plus ou moins considérée comme relevant d'une dynamique psychologique universelle dont le déploiement ou développement tendait vers son but "réaliste".

La seconde approche était à peine esquissée. Elle était très peu connectée à la première et concernait les apprentissages techniques qui accomplissent la dynamique réaliste. Ces savoirs techniques et ces savoir-faire étant appris dans des conditions plus ou moins formelles et réglées, ils n'ont pas intéressé les théoriciens qui les ont abandonnés aux pédagogues, artistes et techniciens.

Il résultait de cette double approche que l'apprentissage de la représentation était conditionné par le développement intellectuel de l'enfant, et qu'il se prolongeait éventuellement dans des apprentissages techniques plus ou moins spécialisés. Nous verrons plus loin qu'il est possible de réinterpréter ces conceptions à la lumière de théories plus récentes.

\subsection{La communication par schémas}

La sémiotique cognitive a permis d'aborder la production des signes graphiques des enfants et des sujets novices sous un angle nouveau ${ }^{1}$. Les expérimentations, les études comparatives et les études sémiotiques ont ainsi permis de montrer que les "schémas" enfantins et novices sont très largement dépendants des opérations cognitives de la conceptualisation, notamment de l'abstraction et de la catégorisation, et, en conséquence, de la sélection socioculturelle des propriétés figuratives des catégories cognitives.

Il apparaît que les compétences figuratives sont acquises dans l'ambiance très ouverte des apprentissages ordinaires qui stabilisent peu à peu les catégories cognitives et qui en figent une sorte de "résumé" destiné à faciliter les échanges verbaux ordinaires, mais aussi les échanges graphiques. Le résultat graphique consiste en une série de schémas plus ou moins standardisés, rapidement exécutables

1 B. DARRAS, $A u$ commencement était l'image, op. cit.; B. DARRAS, "L'image, une vue de l'esprit", Recherches en communication, $\mathrm{n}^{\circ} 10,1998$, pp. 77-99. 
et bénéficiant d'une bonne reconnaissance. Ces pictogrammes sont les ancêtres de tous nos systèmes mnémographiques, mais aussi des systèmes d'écriture, et des systèmes pictographiques.

Dans les usages sociaux ordinaires, et même dans les usages scolaires ces savoirs ne sont jamais explicités, et leur origine n'est généralement pas connue. En l'absence de véritables relais éducatifs, pour l'immense majorité des individus, ils restent des savoirs informels peu exploitables.

Pourtant, un engagement éducatif conscient du potentiel communicationnel pourrait facilement intervenir sur trois niveaux.

- Le premier viserait à promouvoir, valoriser et stimuler les occasions de production graphique dans les échanges interpersonnels entre enfants, entre enfants et adultes, ainsi qu'entre adultes. En termes d'apprentissage, cette approche reposerait essentiellement sur des interactions de communication systématiquement enrichies de schémas et d'images.

- Le second serait beaucoup plus "formel". Il procèderait du souci de perfectionnement et d'accomplissement de la communication intégrant les schémas standardisés. Le projet éducatif serait cette fois destiné à favoriser l'explicitation, la compréhension et la réalisation de ces schémas et de ces pictogrammes. À cette occasion, les "règles" de la communication visuelle seraient "révélées". Cet apprentissage intègrerait de façon pratique et théorique les données de la schématologie et de la sémiotique visuelle.

- Le troisième type d'apprentissage résulterait d'une recherche de complémentarité. Il relève de la conception écosystémique telle que nous l'exposons dans la prochaine partie. Il concernerait la découverte et l'exploration d'autres systèmes de représentation. L'objectif serait autant de protéger le système initial des confusions et contaminations diverses, que d'enrichir le répertoire des systèmes de production d'images.

\subsection{L'approche systémique}

L'approche sémio-systémique traite les différentes familles d'images comme de véritables systèmes de signes disposant de leur 
organisation, de leurs fonctions, de leurs règles, de leur cohérence, de leurs finalités, etc. ${ }^{1}$.

$\grave{A}$ ce titre, les signes produits par les enfants et les adultes novices ne constituent qu'un système parmi les autres. Il s'en distingue toutefois par le fait qu'il est le premier à apparaître. Il se prolonge dans les systèmes pictographiques sophistiqués qui se ramifient en différents systèmes d'écritures visuelles plus ou moins formalisés. D'autres systèmes constituent des savoirs plus ou moins formels tels que la géométrie ou la cartographie qui réclament des apprentissages très formalisés et des systèmes très ouverts qui relèvent du champ artistique.

Dans le système scolaire, plus les systèmes d'images sont formalisés, plus ils relèvent de disciplines consacrées, plus leur apprentissage est systématique et contrôlé. En revanche, plus ils sont ouverts et complexes, moins leur apprentissage est contrôlé. Leur maîtrise dépend alors de la capacité des sujets à l'autodidaxie.

Il faut noter une étrange exception qui concerne les systèmes fortement mimétiques. Bien qu'utilisés dans diverses disciplines scolaires -notamment dans celles qui utilisent le dessin d'observation, (géologie, géographie, biologie, etc.)- leur apprentissage n'est pris en charge par aucune d'entre elles. Tout se passe comme si le modèle piagétien pouvait fonctionner spontanément jusqu'au terme du "réalisme visuel". Bien que l'expérience démente totalement ce mythe, nul ne semble pressé d'intervenir, et les résultats sont bien décevants.

\subsection{L'approche écosystémique}

L'étude des systèmes n'est pas qu'intra-systémique, elle se prolonge dans l'étude des frontières, des liens, des échanges, et des métissages. Cette approche écosystémique permet de modéliser

1 B. DARRAS, Au commencement était l'image, op. cit. ; B. DARRAS, "L'image, une vue de l'esprit", op. cit. ; A.M. KINDLER, B. DARRAS, "Map of Artistic Development", in A.M. KINDLER (ed.), Child Development in Art, Reston, NAEA., 1997, pp. 17-44 ; A.M. KINDLER, B. DARRAS, "Artistic Development in Context : Emergence and Development of Pictorial Imagery in the Early Childhood Years", Visual Art Research, vol. 20, n²2-40, 1994, pp. 1-13 ; F. CASANOVA, B. DARRAS, "Multimédia et métasémiotique iconique", in B. Darras (ed.), Multimédia et savoirs, op. cit., pp. 157-169. 
l'ensemble de la culture visuelle comme un vaste environnement dont certaines zones sont très homogènes et $d$ 'autres moins.

Cette nouvelle approche conduit à penser chaque culture visuelle comme un écosystème composé d'une multitude de systèmes d'imagerie, plus ou moins puissants, plus ou moins valorisés, plus ou moins efficaces, plus ou moins répandus, plus ou moins connus, plus ou moins formels, plus ou moins enseignés, et plus ou moins pratiqués. Mais aussi plus ou moins distincts, plus ou moins étanches et plus ou moins ouverts.

Cette approche permet aussi d'étudier tous les signes résultant de croisements divers non plus comme des monstres incohérents ou ratés, mais comme des signes inter systémiques.

En effet, la majorité des individus ne maîtrise pas les systèmes de signes car ils les pratiquent peu ou n'ont pas été instruits de leurs fonctionnements et de leurs règles. C'est ainsi qu'ils improvisent des bricolages sémiotiques dont les plus fréquents sont un mélange de schémas pré-pictographiques et de similis (c'est ainsi que nous appelons les signes qui visent la ressemblance optique).

En termes de recherche, la focalisation mérite alors d'être décentrée de la cohérence intra-systémique à l'étude des propriétés émergentes, des croisements, des rencontres, des mélanges, des mixités et autres métissages.

Grâce à cette approche, il est possible de réinterpréter les observations et conceptions de Jean Piaget. Alors qu'il croyait observer un processus d'évolution psychique, il observait un processus de métissage et de passage entre deux des plus importants systèmes de signes graphiques. Le système schématique d'une part, et le système mimétique optique d'autre part. Ce qu'il observait n'était donc pas un processus de maturation de l'intelligence, mais les progrès du conflit d'influences culturelles entre deux systèmes de signes et les conflits cognitifs qui en résultent.

Si Piaget avait accordé plus d'attention aux productions des adolescents et des adultes novices, il aurait certainement constaté que les signes n'atteignaient pas la destination qu'il supposait, ce qui lui aurait sans doute permis de réviser son cadre théorique. 


\section{Conclusions}

Les études ici présentées posent à la société un ensemble de questions qui concerne ses engagements dans les processus d'apprentissage et d'intégration des propriétés iconiques et visuo-spatiales dont on a vu la probable influence sur les compétences intellectuelles (De nombreux autres exemples auraient pu être présentés).

L'une des questions les plus urgentes concerne le système d'éducation et sa vocation à promouvoir les connaissances et à réduire les inégalités, y compris dans ce domaine.

En d'autres termes, l'éducation doit-elle prendre en charge ces domaines, ou doit-elle laisser la sélection culturelle opérer au déficit de ceux qui stagnent ou abandonnent, et au bénéfice des autres ?

Dans la mesure où elle parvient à assurer la formation avec un certain succès pour les formes parlées et écrites des langues, ne devrait-elle pas aussi tenter de l'assurer pour les systèmes sémiotiques visuels et iconiques?

Trois enjeux sont pourtant décisifs. Le plus important semble être le développement des formes d'intelligence iconique, visuelle et spatiale.

Le second l'est à peine moins, car il concerne les savoirs et les connaissances nécessaires à la pratique des images et des médias d'image. Enfin, partout où cela est possible, il faudrait aussi tenter de réduire les inégalités de compétence et de connaissance entre les individus, ce qui devrait se faire sans pour autant négliger la recherche d'excellence pour ceux qui manifestent des aptitudes exceptionnelles. 